\title{
Dysautonomia after Spinal Cord Injury: A Clinical Case
}

\author{
Andrade $\mathrm{MJ}^{*}$ and Santos-Jorge, $\mathrm{I}^{*}$
}

Physical and Rehabilitation Medicine Department, Centro Hospitalar Universitário do Porto, Instituto de Ciências Biomédicas Abel Salazar, Porto, Portugal

*Both authors contributed equally

*Corresponding author: Andrade MJ, MD, PhD, SCI Unit, Physical and Rehabilitation Medicine Department, Centro Hospitalar Universitário do Porto, Instituto de Ciências Biomédicas Abel Salazar, Porto, Portugal, E-mail: mjandrade@icbas.up.pt

Citation: Andrade MJ, Santos-Jorge, I (2018) Dysautonomia after Spinal Cord Injury: A Clinical Case. J Case Rep Stud 6(5): 504. doi: 10.15744/2348-9820.6.504

Received Date: July 21, 2018 Accepted Date: October 29, 2018 Published Date: October 31, 2018

\begin{abstract}
Spinal cord injury (SCI) causes not only motor and sensory deficits but also autonomic dysfunctions. Sweating disorders are frequent following spinal cord injury, the most common being hyperhidrosis.

The following case report describes hyperhidrosis secondary to fecal impaction with intestinal distension and indwelling catheter of progressively larger caliber and how physical and rehabilitation medicine improved the patient's quality of life.

Although dysautonomia is a manifestation of the acute phase of spinal cord injury and autonomic dysreflexia is a manifestation of the chronic phase, our patient, in the chronic phase, had dysautonomia with profuse sweating and no dysreflexia, as would have been expected. The absence of significative blood pressure changes allowed the patient to survive without severe sequelae.

This case report confirms the importance of treatment of SCI patients in spinal units (as in most of the world) where knowledge of SCI complications allows for easy diagnosis and earlier treatment.

Keywords: Autonomic Dysfunctions; Hyperhidrosis; Spinal Cord Injury
\end{abstract}

\section{Introduction}

Spinal cord injury (SCI) is one of the most devastating injuries both physically and psychologically. It causes not only motor and sensory deficits but also autonomic dysfunctions [1,2].

The impairment of connections between superior brain centers and the spinal cord causes several autonomic abnormalities that can affect the cardiovascular, respiratory, urinary, gastrointestinal, thermoregulatory and sexual systems [3,4].

Sweating disorders are frequent complaints of SCI. The most common, in response to noxious stimuli below the level of the SCI, is hyperhidrosis. Hyperhidrosis, or excessive sweating, can be accompanied by significant social and emotional distress, negatively affecting an individual's quality of life. The symptom can be associated with other more serious conditions such as autonomic dysreflexia or can exist on its own [5].

The following case report describes dysautonomia with hyperhidrosis and how physical and rehabilitation medicine improved the patient's quality of life. In fact, although dysautonomia is a manifestation of the acute phase of spinal cord injury and autonomic dysreflexia is a chronic phase, our patient, in the chronic phase, had a dysautonomia with profuse sweating and no dysreflexia, as would have been expected.

The autonomic dysfunctions of spinal cord injuries aren't well known, in general, by physicians. So it is very important that the treatment is done in specializes units.

\section{Case Report}

A 57-year-old woman with a history of C4 ASIA Impairment Scale A tetraplegia, since 1980. Treated at the time at a Central Hospital and a Rehabilitation Center. After that, no follow-up in specialized SCI Unit.

In 2013, the patient was directed to an internal medicine consultation through general and family medicine for hyperhidrosis with 1 
year of evolution. The patient was admitted to the Department of Internal Medicine, where endocrinological (pheochromocytoma, hyperthyroidism), neoplastic (carcinoid, renal cell neoplasia, VIPoma or thyroid carcinoma) and mastocytosis pathologies were excluded. After these multiple diagnostic procedures, the patient was discharged without treatment or a defined plan and with continuation of the complaints. Subsequently, after attending several specialty appointments (internal medicine, vascular surgery, endocrinology), the patient was directed to a physical medicine and rehabilitation consultation in 2014.

The first time we saw the patient she was hemodynamically stable, apyretic and normotensive, with profuse sweating (face and neck) and significant abdominal distension. She had an indwelling catheter (but still had urine losses), and irregular bowel movements without medication. The abdominal-pelvic computed tomography (CT) scan showed distension of the intestine loops with gas and feces but did not reveal any specific cause of obstruction. This suggested that we were facing a SCI with autonomic changes for two important stimuli: fecal impaction with intestinal distension due to the absence of effective intestinal training and an indwelling catheter of progressively larger caliber.

We adjusted the diet, started medication (senna and contact laxative) to promote regular intestinal transit, reduced the size of the indwelling catheter and prescribed an anticholinergic (oxybutynin) to reduce the hyperreflexic response to the presence of the catheter. The patient's condition improved tremendously. The abdominal distension disappeared and sweating was restricted to the left hemiface. She maintains an indwelling catheter without incontinence and her intestinal transit has become regular. Follow-up has been maintained in the last 4 years.

\section{Discussion}

Although dysautonomia is a manifestation of the acute phase of spinal cord injury and autonomic dysreflexia is a manifestation of the chronic phase, our patient, in the chronic phase, had dysautonomia with profuse sweating and no dysreflexia, as would have been expected. The absence of significative blood pressure changes enabled the patient to survive without severe sequelae.

Clinical stabilization was possible with regularization of intestinal transit and introduction of anticholinergic (oxybutynin). Oxybutynin is one of the anticholinergics most used in Portugal because of its efficacy, low cost and tolerability among the population with spinal cord injury.

It is extremely important to recognize autonomic dysfunctions as a consequence of noxious stimulus after a spinal cord injury, since this is a potentially life-threatening condition.

Physicians from other specialities should pay attention to SCI patients and reference them to Physical and Rehabilitation Medicine. The follow-up at a SCI outpatient unit is essential for these patients in order to prevent risk behaviors and to ensure an early detection of autonomic dysfunction and their appropriate treatment.

\section{References}

1. Roque V, Cunha I, Rocha A, Andrade MJ (2013) Autonomic Nervous System Dysfunction after Spinal Cord Injury. Revista da Sociedade Portuguesa de Medicina Física e de Reabilitação 24: 43-51.

2. Krassioukov AV, Karlsson AK, Wecht JM, Wuermser LA, Mathias CJ, et al. (2007) Assessment of autonomic dysfunction following spinal cord injury: rationale for additions to International Standards for Neurological Assessment. J Rehabil Res Dev 44: 103-12.

3. West CR, AlYahya A, Laher I, Krassioukov A (2013) Peripheral vascular function in spinal cord injury: a systematic review. Spinal Cord 51: 10-9.

4. Walters ET (2012) Nociceptors as chronic drivers of pain and hyperreflexia after spinal cord injury: an adaptive-maladaptive hyperfunctional state hypothesis. Front Physiol 3: 309 .

5. Hou S, Rabchevsky AG (2014) Autonomic consequences of spinal cord injury. Compr Physiol 4: 1419-53.

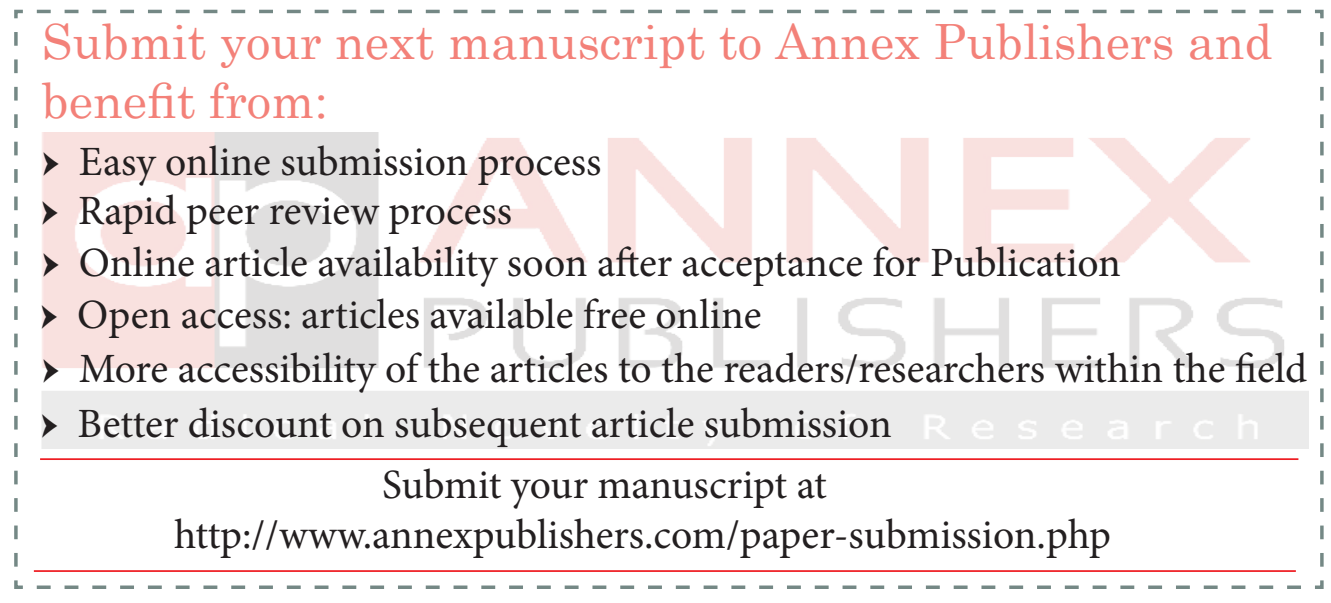

